\title{
Una aproximación poliédrica al concepto microeconómico
}

\author{
Penélope Hernández Rojas \\ Profesora Titular del Departament d'Anàlisi Econòmica de la Universitat de València \\ penelope.hernandez@uv.e
}

José E. Vila Gisbert

Profesor Titular del Departament d’Anàlisi Econòmica de la Universitat de València jose.e.vila@uv.es

|Fecha presentación: 13/09/2009 | Aceptación: 16/11/2009 |Publicación: 04/12/2009

\begin{abstract}
Resumen
Este artículo propone un enfoque tridimensional en el aprendizaje de cualquier concepto en microeconomía. Las tres facetas del aprendizaje presentadas son la experiencial- vivencial, la gráfico-geométrica y la matemático-formal. En cada una de ellas el lenguaje utilizado es diferente, y el paso entre las tres facetas conforma el camino apropiado para la adquisición profunda del concepto microeconómico a estudio. Se incorporan, además, dos ejemplos, donde se ilustra el método de la aproximación poliédrica de dos conceptos microeconómicos estándar en los programas de Introducción de la Microeconomía y Microeconomía Intermedia.
\end{abstract}

Palabras clave: didáctica de la microeconomía, método de caso, faceta experiencial-vivencial, faceta gráfico-geométrica, faceta matemático-formal

\begin{abstract}
Resum
Aquest article proposa un enfocament tridimensional en l'aprenentatge de qualsevol concepte en microeconomia. Les tres facetes de l'aprenentatge presentades són l'experiencial- vivencial, la grafico-geomètrica i la matematico-formal. En cada una d'elles el llenguatge utilitzat és diferent, i el pas entre les tres facetes conformen el camí apropiat per a l'adquisició profunda del concepte microeconòmic a estudi. S'incorporen, a més, dos exemples, on s'illustra el mètode de l'aproximació polièdrica de dos conceptes microeconòmics estàndard en els programes d'Introducció a la Microeconomia i Microeconomia Intermèdia
\end{abstract}

Paraules clau: didàctica de la microeoconomia, mètode de cas, faceta experiencial-vicencial, faceta gràfico-geomètrica, faceta matemàtica-formal

\begin{abstract}
This paper proposes a three-dimensional approach to learn any microeconomics concept. There are three learning facets called the experience-living facet, the graphic-geometric facet, and the mathematical-formal facet. The language used in each facet is different and the conexion among them conforms the right way to achieve a deeply understanding of the targeted microeconomics concept. Two practical examples are presented where we illustrate the methodology of the polyhedra approximation for two microeconomics concepts in a standart program of Introduction of Economics and Microeconomics.
\end{abstract}

Keywords: microeconomics didactics, case methodology, experience-living facet, graphic-geometric facet, mathematical-formal facet 


\section{Introducción}

Un objetivo esencial de cualquier curso universitario de microeconomía es capacitar al estudiante para que llegue a razonar, con un nivel alto de abstracción, sobre situaciones que le son familiares, tanto por su propia actividad como agente económico (consumidor y tal vez trabajador o emprendedor) como por la información que fluye continuamente hasta él referente al comportamiento de los mercados, actuaciones de empresa, programas de política económica, etc. En otras palabras, en microeconomía se modelizan, recurriendo a herramientas relativamente sofisticadas, facetas de la realidad de las que el alumno tiene una experiencia vivencial cercana, y sobre las que el estudiante se ha formado previamente, y muchas veces de modo irreflexivo, opiniones e incluso ideologías que pueden estar fuertemente arraigadas. Por ejemplo, en un curso de microeconomía se plantean modelos relativamente formalizados que intentan explicarle al alumno sus propias decisiones de compra al visitar un supermercado.

En este contexto, un estudiante puede fácilmente llegar a pensar que la microeconomía no le dice nada nuevo que él no pueda experimentar por si mismo y de forma mucho más directa. Incluso, cuando los razonamientos microeconómicos le lleven a conclusiones que no se había planteado o contradigan sus pre-conceptos, puede llegar a pensar que esa construcción artificiosa es totalmente falsa o creada con fines manipuladores. Como consecuencia de estos hechos, puede producirse el fenómeno de que el alumno tenga la sensación de que la microeconomía no es más que un sistema formal, creado con unos conceptos puramente academicistas, interesante (en el mejor de los casos) por sí mismo, pero carente de conexiones con la economía real. En este caso, el estudiante niega, consciente o inconscientemente, a la microeconomía todo valor como herramienta para la comprensión y predicción de la realidad, con lo que esta se convierte en una disciplina ajena a la misma: es lo que denominamos alienación de la teoría ${ }^{1}$. Cualquier diseño de planteamiento didáctico debe estudiar en qué puntos del proceso de enseñanza-aprendizaje puede producirse dicha alienación, cuáles son los motivos concretos por los que puede producirse, y cómo evitar que finalmente ocurra esta alienación de la teoría.

En este trabajo planteamos una teoría para comprender las razones por las que esta alienación llega a producirse, basada en una aproximación poliédrica a los conceptos microeconómicos. Dicha teoría proporciona indicaciones para el desarrollo de estrategias de enseñanza-aprendizaje en microeconomía, centradas en el tránsito entre las diferentes facetas que conforman un concepto microeconómico y que ayuden a evitar este fenómeno de alienación.

$\mathrm{El}$ artículo se estructura de la siguiente forma: el apartado 2 plantea las ideas relativas al carácter multifacético del concepto microeconómico, e introduce una definición y nomenclatura precisas de sus distintas facetas. En el apartado 3 se aborda la cuestión fundamental del tránsito entre facetas y la dificultad de efectuar algunos de estos tránsitos como causa última del fenómeno de alienación de la teoría.
El apartado 4 presenta algunas implicaciones de estas ideas en el proceso de aprendizaje-enseñanza de la introducción a la microeconomía y la microeconomía intermedia. Por último, el artículo se cierra con dos casos prácticos muy sencillos utilizados por los autores para desarrollar en los alumnos habilidades de tránsito entre las distintas facetas de un concepto.

2. Naturaleza poliédrica del concepto microeconómico La aproximación a cualquier concepto microeconómico puede hacerse desde diferentes puntos de vista. Por ejemplo, si consideramos el concepto de relación marginal de sustitución técnica (RST de ahora en adelante) de capital por trabajo en una empresa, este concepto puede ser entendido simultáneamente al menos cuatro formas:

1. La tasa a la que se puede reducir el capital agregando más trabajo en esta empresa para que el nivel de producción no cambie.

2. La pendiente de una curva (isocuanta asociada al nivel actual de producción) en un punto (el correspondiente a los niveles actuales de capital y trabajo de la empresa). 3. La derivada de una función (la función capital, con variable independiente trabajo) respecto de su variable independiente (trabajo), en un punto (nivel actual de trabajo).

4. El cociente de dos derivadas (productos marginales) en un punto (el correspondiente a los niveles actuales de trabajo y capital).

Así, la acepción uno parece no estar asociada con ningún tipo de modelización ${ }^{2}$, sino que enuncia algo que cualquier agente productor puede haber vivido: tener una avería en máquina y tener que calcular cuantos trabajadores adicionales necesita contratar para cumplir con sus compromisos de producción adquiridos. Se trata, pues, de una acepción vivencial ${ }^{3}$ del concepto. Por otro lado, la acepción dos se plantea en términos puramente geométricos (pendiente en un punto), mientras que las tres y la cuatro se enuncian en términos tomados del cálculo básico (funciones y derivadas) y, utilizando manipulaciones algebraicas, es posible ver que son equivalentes. Tenemos, pues, que el concepto microeconómico de RST tiene tres acepciones o, como preferimos denominar, facetas distintas: es pues un concepto poliédrico ${ }^{4}$

El carácter poliédrico del concepto microeconómico tiene dos implicaciones clave en el proceso de enseñanzaaprendizaje de la microeconomía:

- Para estudiar un mismo concepto resulta necesario utilizar diferentes esquemas de razonamiento (concretados en diferentes lenguajes), cada uno de ellos relacionado con una faceta de dicho concepto.

- Un concepto microeconómico no está asumido cuando se conocen todas o algunas de sus facetas, sino cuando se dominan las relaciones o -como denominaremos de ahora en adelante- tránsitos entre ellas.

\footnotetext{
${ }^{1}$ El concepto de alienación de la teoría es un concepto propio de los autores para definir la dicotomía entre teoría y práctica.

${ }^{2}$ En la acepción uno está implícito, desde luego, un modelo, aunque no de carácter formal.

${ }^{3}$ Hemos descartado otras denominaciones para esta faceta como la de real o económica, aunque esta acepción no es más real ni económica que las otras tres.

${ }^{4}$ Análisis más detallados permitirían definir más facetas del concepto (como la numérico-computacional), pero para los objetivos de este trabajo resulta más adecuado limitarse a las tres dimensiones básicas que constituyen el concepto.
} 


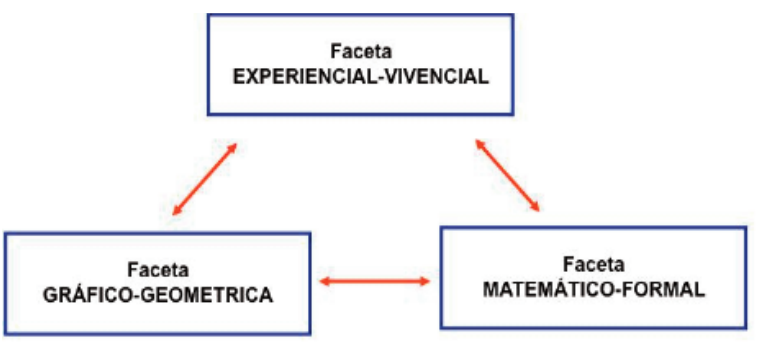

Figura 1: Facetas de un concepto microeoconómico

En general, dado cualquier concepto microeconómico, estableceremos tres facetas diferentes a las que denominaremos con una combinación de dos términos: el primero referido a los elementos en que se expresa el concepto en esa faceta (vivencias / elementos gráficos / elementos algebraicos) y el segundo al tipo de razonamiento válido en cada faceta (natural / geométrico / formal). La diferenciación entre los tipos de lenguaje-razonamiento en cada faceta está relacionado con el análisis de la evolución de los niveles de racionamiento y lenguajes del proceso de aprendizaje-enseñaza de la geometría introducidos por van Hiele (1986). Dicho método ha sido aplicado en la disciplina de matemáticas, como así lo demuestran los trabajos de Jaime (1993), Jaime y Gutiérrez (1994) y Peeg, Gutiérrez y Huerta (1997) entre otros, en la adecuación del modelo de van Hiele y la enseñanza de geometría ${ }^{5}$ euclídea. Así, tenemos (Figura 1):

- Faceta vivencial-natural: recoge la imagen que tendría de él un agente económico sin formación geométrica o matemática, que reflexionase racionalmente, de una forma lógica y precisa, sobre el aspecto de la realidad económica a la que éste se refiere. Tanto la definición del concepto como sus propiedades se realizan en lenguaje natural, sin referencia a elementos gráficos o algebraicos. Si bien en los razonamientos no se recurre a elementos manipulativos de carácter geométrico o formal, los procesos de deducción son concretos y rigurosos, dando lugar a predicciones contrastables, si bien éstas serán habitualmente de carácter más bien cualitativo. Esta aproximación al concepto es en la que el alumno suele sentirse más cómodo y cercano a la realidad.

- Faceta gráfico-geométrica: el concepto viene dado por una colección de elementos geométricos (curvas como isocuantas o curvas de indiferencia, puntos cuyas coordenadas son el nivel de capital y trabajo de una empresa, etc.), o como una propiedad o relación geométrica entre ellos (pendientes, puntos de corte, condiciones de tangencia, etc.). El razonamiento es ahora de carácter geométrico $\mathrm{y}$, a diferencia de lo que sucedía en la aproximación vivencial-natural, se llega a obtener conocimiento sobre la realidad económica con deducciones que involucran elementos puramente gráficos.
- Faceta matemático-formal: el concepto microeconómico se plantea en términos de elementos matemáticos (funciones, conjuntos, variables, etc.), y sus propiedades (derivada de una función, solución de un sistema de ecuaciones, etc.), tomados habitualmente del cálculo, álgebra, investigación operativa, estadística y teoría de juegos. El tipo de razonamiento en esta faceta es el matemático-formal. Los conceptos se definen formalmente, se establece una axiomática precisa y, a partir de aquí, por medio de demostraciones rigurosas, se establecen conclusiones y se hacen predicciones de carácter cuantitativo y cualitativo sobre los comportamiento de los agentes, que pueden ser contrastadas empíricamente con técnicas estadísticas, experimentales o econométricas.

3. El tránsito entre facetas y la alienación de la teoría

Un concepto microeconómico no está asimilado todavía en el momento en que se interiorizan sus tres facetas. Es necesario asimilar también que éstas constituyen aspectos complementarios y dependientes de un mismo concepto, y que el alumno sea así capaz de relacionar y combinar de forma correcta los tres tipos de razonamiento. Es lo que denominamos como el tránsito entre facetas.

Cuando el alumno no puede transitar entre facetas aparecen una serie de problemas que dificultan la asimilación del concepto:

- No se percibe un solo concepto, sino dos o tres conceptos distintos, como mucho compartiendo un mismo nombre. - Las facetas gráfico-geométrica y matemático-formal se alienan, se separan de la realidad y se convierten en construcciones carentes de verdadero significado y de utilidad para comprender la realidad económica.

- Se emplea el razonamiento válido en una faceta para manipular elementos de otras.

Es importante destacar que los tránsitos entre facetas no pueden describirse mediante las reglas de razonamiento de ninguna de ellas en particular, sino que es necesario recurrir a un meta-razonamiento que englobe y trascienda a los otros tres. Capacitar para la realización efectiva de estos tránsitos constituye, en nuestra opinión, uno de los retos fundamentales para la didáctica de la microeconomía.

El marco teórico presentado permite definir, con precisión, el concepto de alienación de la teoría como la incapacidad del alumno para transitar entre la faceta experiencial-vivencial y las otras dos facetas que constituyen cada concepto.

Esta situación es muy frecuente en el aula de microeoconomía, como revela por ejemplo el trabajo empírico expuesto en Vila (2004) ${ }^{6}$. En este trabajo se contrastó que el alumno tiende a identificar el concepto con su faceta vivencial-natural, no viendo el interés ni la necesidad de las otras dos:

“...me agobian mucho las matemáticas, sobre todo si no hay números. No sé qué tiene que ver todo eso con el mundo real..."

\footnotetext{
${ }^{5}$ La fuerte utilización del método geométrico en microeconomía nos da rigor en la utilización de esta técnica pedagógica, diseñada en un principio para explicar la geometría.

${ }^{6}$ En este trabajo se analizó la visión del estudiante acerca de cada uno de los componentes del modelo didáctico utilizado para la enseñanza de la microeconomía en su universidad, a fin de detectar cuáles son los obstaculizadores y facilitadores que se presentan en el proceso de enseñanza-aprendizaje de la microeoconomía. Dicho estudio se emprendió desde una óptica cualitativa. El instrumento de recogida de información escogido fue la entrevista en profundidad a doce alumnos de las carreras de ADE y Economía de la Universidad de Valencia (España) y la Universidad Nacional de Mar del Plata (Argentina), que hubieran cursado una asignatura de Microeconomía Intermedia durante el curso 2003-2004.
} 
“...los gráficos son muy complicados, con tantos puntos y tantas líneas.... al final te los aprendes de memoria y los pones en el examen, pero no sabes para qué los haces ni lo que quieren decir...."

“... el gráfico no ayuda a entender (el concepto), has de entender las dos cosas por separado...”

Por otro lado, el estudiante presta tanta atención a los detalles de la manipulación algebraica o al trazado de gráficos, que se olvida de la faceta vivencial-natural:

“... lo importante en el examen es no equivocarte en un signo y que el problema dé bien. No importa tener claras las ideas o no si sabes como calcular..."

"... en clase estoy más pendiente de las operaciones que de otras cosas ...”

La alienación surge, en su opinión, de la excesiva abstracción y simplicidad de los modelos considerados, lo que les dificulta el tránsito a la etapa experiencial-vivencial:

"¿Dónde podemos encontrar un mercado que sea perfectamente competitivo? Eso no existe...."

"...lo que más me fastidia es la cantidad de supuestos que hacemos para que así salgan cosas: que si una empresa sabe los costes de la otra, que si la función de demanda es lineal... y luego, con muchos cálculos sacamos conclusiones que queremos aplicar a la realidad, como que los mercados son eficientes y que mejor que el gobierno no haga nada...."

\section{Implicaciones sobre el proceso de enseñanza-apren-} dizaje de la microeconomía

Según lo discutido anteriormente, uno de los retos clave en el aula es conseguir que el alumno asimile un concepto microeconómico en sus tres facetas y pueda transitar entre ellas, evitando que, en el mejor de los casos, llegue a asimilar dos o tres conceptos diferentes:

- El concepto vivencial-natural, por un lado: lo que los consumidores o las empresas hacen de verdad en el mundo real o cómo funcionan de verdad los mercados - Un (o en algunos casos dos) concepto(s) de carácter gráfico-geométrico o matemático-formal, que comparten el mismo nombre que el vivencial-natural pero que, en el fondo, no lo representan ni ayudan a comprenderlo, sino que lo alienan.

Si se llega a producir esta separación, se genera un rechazo del estudiante respecto a la asignatura, debido a su sensación de estar perdiendo el tiempo o de incumplimiento de sus expectativas de lo que suponía que era estudiar economía. La falta de integración crea la imagen de la microeconomía como algo separado de la "economía de verdad" (es decir, la faceta vivencial-natural) y que no puede ayudar a conocer, mejorar o predecir ésta. Según Vila (2004), esta separación se produce muchas veces ya en los conceptos analizados en las primeras clases (preferencias / utilidad / curvas de indiferencia), y se va ampliando conforme se introducen nuevos conceptos basados en éstos y que se suponen integrados por el estudiante. El desarrollo de metodologías y técnicas didácticas que transiten entre facetas resulta, pues, necesario desde las primeras clases.
Dentro de las posibles herramientas didácticas, la utilización de casos puede jugar un papel clave en la capacitación para transitar entre facetas. El objetivo de un caso es muy diferente al de un ejemplo. El caso no proporciona soluciones, sino datos concretos para que el alumno reflexione y plantee posibles soluciones, que luego se puedan discutir dentro de un enfoque de aprendizaje activo. Para ser realmente efectivo (López 1997) un caso debe ser breve, orientado a las características y experiencias del grupo concreto de estudiantes que lo utiliza, y con preguntas abiertas.

\section{Ejemplos de trabajo de tránsito entre facetas en el aula} En esta siguiente sección se plantean dos casos que se han utilizado con éxito en un curso de Microeoconomía Básica (Hernández 2009) e Intermedia (Vila 2009) y que se presentaron en las Primeras Jornadas de Docencia en Microeoconomía, celebradas en Granada en 2009.

5.1. El tránsito entre las facetas gráfico-geométrica a la matemático-formal: del concepto de medio al marginal - La tableta de chocolate

Dos de los conceptos más utilizados en la formalización de decisiones en microeconomía son los conceptos de valor medio y marginal. Si bien estos conceptos pueden encontrarse a menudo en una faceta experiencia-vivencial de los alumnos, resulta a veces complicado transitar en un curso de introducción a la microeconomía a su faceta geométrica y, por último, a la analítica. Cuando se explica a los alumnos la influencia de la última unidad en el ejercicio productivo, éstos suelen entender sin demasiada dificultad como ésta modifica los valores totales y en los medios (el clásico ejemplo de las notas medias respecto a la última nota de esa asignatura es uno de los muchos ejemplo utilizados para explicar la relación entre estos conceptos). Sin embargo, la competencia indispensable en un primer curso de microeconomía de adecuar dichos conceptos al plano geométrico no es, en general, sencilla de desarrollar. En nuestro lenguaje, estaríamos hablando de pasar de una faceta experiencia-vivencial a una faceta gráfico-geométrica de los conceptos de valor marginal y medio y de su relación. Normalmente, el método utilizado para conseguir este objetivo es utilizar la representación de funciones reales, es decir, se pasa directamente a una notación (fase) analítica, lo que hace aparecer muy frecuentemente el fenómeno de la alienación de la teoría. Este apartado propone un ejemplo tomado de Hernández (2009), donde se utiliza un entorno geométrico donde se pueden entender los conceptos en este medio. Nos encontramos, pues, en la faceta gráfico-geométrica.

Para empezar, tomamos como base el área de un rectángulo para cuantificar, por ejemplo, el total del coste de pro-

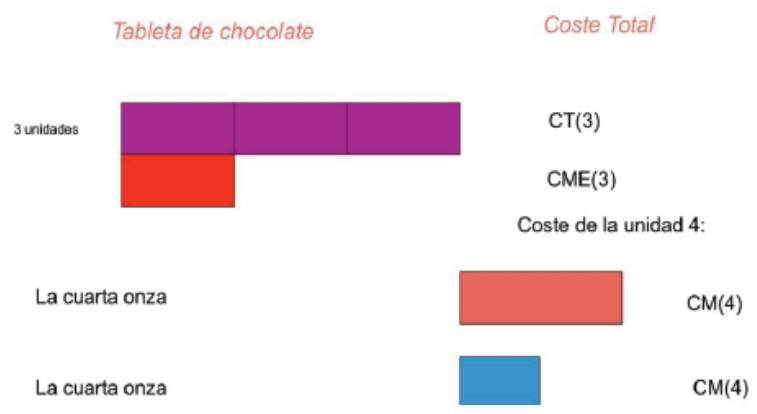

Figura 2: Representación gráfica de los costes de producción medio y marginal 


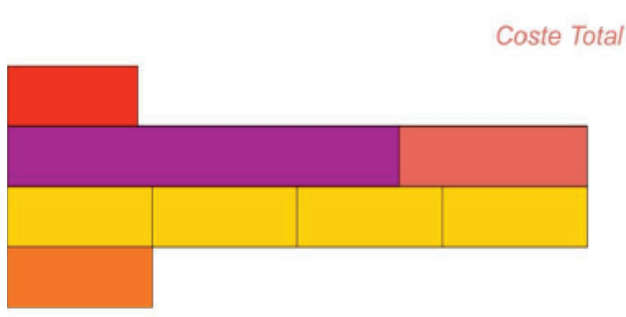

$\mathrm{CT}(4)$

Figura 3: Representación gráfica de la relación de los costes medio y marginal (coste medio creciente)

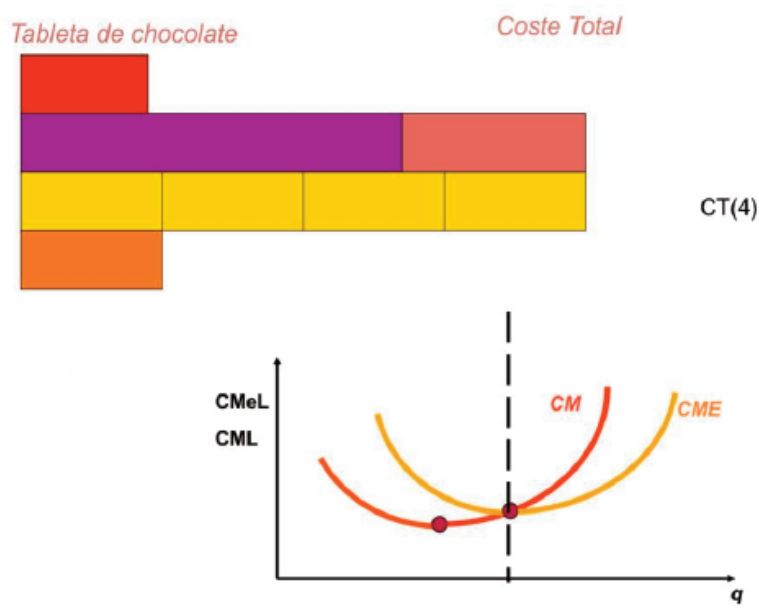

Figura 4: Representación gráfica de la relación de los costes medio y marginal (coste medio creciente)

ducción. Así, considerando que se producen tres unidades, podemos cuantificar el coste medio, es decir, el valor medio de cada unidad. Basta con dividir el rectángulo en tres partes iguales, y cada trozo representaría el coste medio. Así, tenemos una tableta de chocolate que representa el coste total, y una onza nos representaría el coste medio. La Figura 2 muestra ambos valores espaciales como las áreas vinculados a cada concepto.

Ahora incluimos un nuevo rectángulo, que marca el valor del coste de producción de la cuarta unidad. Este rectángulo puede medir más, igual o menor área que el rectángulo asociado al coste medio. Para cada caso, estudiaremos cómo influye el agregar una nueva unidad en los cómputos del coste total y medio resultante para cuatro unidades.

Empecemos con el caso en el que el coste marginal es mayor que el coste medio, es decir, el coste marginal de la cuarta unidad o el área del rectángulo de color marrón es más pequeño que el área del rectángulo de color rojo que obtuvimos dividiendo el coste total, es decir, un trozo de la tableta de chocolate.

$\mathrm{Al}$ añadir el coste marginal obtenemos un nuevo rectángulo, que corresponde al coste total de cuatro unidades de producción. Tenemos que calcular en nuestra nueva tableta el área correspondiente a cada unidad, dividiéndolo en cuatro porciones que son el coste medio de cuatro unidades. Nos resulta, pues, el coste medio de cada unidad, que corresponde con el rectángulo de color amarillo. La parte inferior de la Figura 3 contiene el coste medio de cuatro unidades como un rectángulo de color naranja. Así, ahora con los tres rectángulos siguientes (el rojo, el marrón y el naranja) podremos interpretar la relación entre los tres conceptos: al añadir el coste marginal de la cuarta unidad (el rectángulo marrón), que es mayor que el coste medio de tres unidades (el rectángulo rojo), el coste

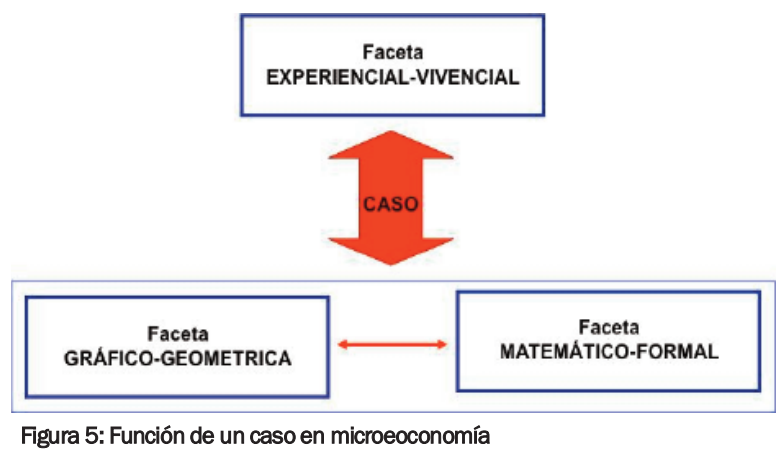

medio resultante crece (esto se aprecia en que el área del nuevo rectángulo resultante naranja es mayor que el rojo anterior).

Por último habría que pasar a la faceta matemático-formal. Con la argumentación anterior se explica en qué región de la gráfica siguiente se representaría el diagrama de los rectángulos anteriores. El crecimiento de la curva de coste medio nos confirma que el coste marginal tiene que ser mayor, por tanto dicha curva debe estar por encima de la anterior (Figura 4)

5.2. El tránsito desde lo vivencial-natural a las otras facetas

Un caso resulta de especial interés para transitar desde la faceta experiencial-vivencial a las otras de carácter más abstracto (Figura 5).

En el siguiente caso (Vila 2009), estilización de un hecho real, se presenta al alumno una serie de cuadros con información que conduce en el aula a una conclusión de carácter muy intuitivo que resulta, tras un análisis más formal, incorrecta. Además de ayudar a desarrollar las facetas gráfico-geométrica y matemático-formal de los conceptos de costes, y la faceta experiencial-vivencial de la condición formal de maximización de beneficios, ejemplos como este son de gran utilidad para generar en el alumno el convencimiento de que el razonamiento intuitivo puede inducir a errores costosos en la toma de decisiones económicas reales, y de que la formalización geométrica o matemática puede aportar valor a la hora de tomar estas decisiones. A continuación vamos a definir el caso.

A. Información de partida. Una escuela de idiomas imparte docencia de apoyo a grupos de alumnos de enseñanza secundaria obligatoria (ESO) y bachillerato. Por las restricciones horarias de los alumnos, que asisten a clases en centros reglados, éstos solo pueden acudir a los grupos que el centro organiza en horario de tarde. La escuela está abierta todo el día para dar información, desarrollar tareas administrativas e impartir algún curso de formación ocupacional. Esto hace que un gran número de aulas estén vacías en el horario de mañana, por lo que el director se plantea ofrecer clases de idiomas personalizadas (a un solo alumno) para personal de diferentes empresas.

$B$. La reflexión del director. Dividiendo el coste anual de alquiler, publicidad, salarios del personal de gestión, etc. entre el número de horas impartidas (alrededor de 12.000 anuales), obtiene un coste del aula de 30 euros por hora. Como un profesor de idiomas cobra 20 euros por hora de clase impartida, el coste total una clase individual de idiomas es de 50 euros por hora. A partir de un pequeño estudio de mercado, el director ha podido determinar que el precio máximo que pagaría un alumno por una hora de clase individual de idioma es de 25 euros por hora.

La reflexión del director es la siguiente: como el coste es de 50 euros por hora y el precio máximo por el servicio es de 25 euros por hora, cada hora de clase individual hace perder al cen- 
tro 25 euros. Así, el servicio no es rentable y es mejor no ofrecerlo.

C. Discusión del caso. La cuestión que se plantea a los alumnos es la evaluación de la decisión tomada por el director. En la experiencia de los autores, casi la totalidad de los alumnos considera en un primer momento que este razonamiento es adecuado. A partir de aquí, y apoyándose (si los hay) en aquellos alumnos que consideran inadecuada la conclusión del director (y que habitualmente no saben justificar por qué), el profesor actúa como moderador de una discusión en la que el alumno llega a darse cuenta por sí mismo de que:

- Mantener el centro cuesta 3x12.000 $=36.000$ euros, se impartan o no clases individuales

- No se han comparado realmente los costes y los ingresos con y sin una clase individual: la diferencia de costes entre no dar o dar la clase es de 20 euros, mientras que la diferencia de ingresos entre no dar o dar la clase es de 25 euros - De esta forma, impartir una clase individual proporciona 5 euros de beneficio extra a la escuela.

D. Formalización gradual del concepto: ¿por qué aumenta el beneficio una clase individual? El punto clave en la formalización está en la introducción gradual de una nomenclatura y unas definiciones precisas de los elementos que aparecen en el razonamiento anterior, de forma que el alumno interiorice que este paso resulta necesario para evitar tomar decisiones incorrectas en la gestión de una empresa. Los elementos clave que resulta necesario formalizar en este caso son las cantidades que han aparecido en el razonamiento como costes e ingresos de la empresa. Concretamente:

-20 euros: incremento de coste por dar la clase (coste marginal)

-36.00o euros: coste de tener abierta la escuela (coste fijo a corto)

-30 euros: coste por hora de tener abierta la escuela (coste fijo medio)

-50 euros: coste aproximado por hora de dar la clase individual (coste medio)

-25 euros: precio del servicio (ingreso marginal)

A partir de aquí, resulta sencillo que el alumno sea consciente por él mismo, a través del proceso de discusión dirigida del caso, que dar una hora de clase incrementa el beneficio del centro, porque 25 euros es más que 20 euros, es decir, que es posible incrementar el beneficio porque el ingreso marginal por una hora de clase es mayor que el coste marginal de impartirla.

Si se pide al alumno que discuta lo que hubiese sucedido si el precio de la clase impartida fuese menor que su coste marginal, se llega de forma intuitiva a la conclusión de que el beneficio de la empresa no puede incrementarse (es máximo) cuando el coste marginal y el ingreso marginal coinciden.

E. Tránsito por las facetas abstractas del concepto. Una vez formalizados los elementos de la decisión, es muy sencillo que el alumno interiorice la abstracción gráfico-geométrica y matemático formal de la maximización de beneficios y, lo que es más importante, transite de nuevo de éstas a la faceta expe- riencial-vivencial, teniendo consciencia de que este proceso ha evitado un error que él mismo podría haber cometido en el desempeño de un trabajo, si no hubiese razonado en términos de coste e ingreso marginal y de sus relaciones.

\section{Conclusiones}

La presencia de alienación con la teoría económica es un hecho constatable que se acentúa en los primeros cursos de grado. Esto es debido a la formación del estudiante medio de la Facultad de Economía (vease Hernández y Pastor 2009) y, además, a la naturaleza de la materia. En este artículo hemos desgranado las distintas partes de cualquier situación que un estudiante se podría encontrar en la asignatura de Microeconomía I dando un patrón de actuación para adecuar el lenguaje y el nivel de comprensión. La experiencia de los autores en la enseñanza de esta asignatura en los últimos cuatro años confirma el éxito de la metodología. En particular, en el curso de primero de Economía del grupo del Proyecto de Innovación Educativa (PIE), el 85\% de los alumnos han aprehendido, siguiendo el primer caso del artículo, los conceptos de medio y marginal, en contraste con el 40\% de los alumnos que han seguido una metología alternativa. Respecto al segundo caso presentado en el artículo, lo más notable es la comprehensión de los conceptos microeconómicos en la decisión real. Este proceso de aprendizaje permite, pues, estimular todas las facetas del aprendizaje.

\section{Bibliografía}

Hernández, P. (2009). Del medio al marginal: la tableta de chocolate. $1^{a}$ Jornada de Docencia de Microeconomía de la Universidad de Granada.

Hiele, Pierre M. van (1986). Structure and insight: a theory of mathematics education. Orlando. Academic Press.

Huerta, M.P. (1997). Los niveles de van Hiele en relación con la taxonomía y los mapas conceptuales. Tesis doctoral. Universidad de Valencia.

Jaime, Adela (1993). Aportaciones a la interpretación y aplicación del modelo de van Hiele: La enseñanza de las isometrías de plano. La evaluación del nivel de razonamiento. Tesis doctoral. Universidad de Valencia.

Jaime, Adela; Gutiérrez, Angel (1994). A model of Test Design to assess the van Hiele Levels. Proccedings of the 18th Conference of the International Group for the Psychology of Mathematics Education, vol. 3, pp. 41-48.

López, Alfonso (1997). Iniciación al análisis de casos. Bilbao: Mensajero.

Peeg, J., Gutiérrez, A, y Huerta, M.P. (1997). Assessing Reasoning Abilities in Geometry, en Carmelo Mammana; Vinicio Villani(eds.) (1998). Perspectives on the teaching of Geometry for the 21st Century. Dordrecht: Kluwer Academic Press, pp.275-295.

Vila, José E. (2004). Proyecto docente para las pruebas de Habilitación Nacional para el cuerpo de Profesores Titulares de Universidad.

Vila, José E. (2009). Caso para la introducción de la condición de maximización de beneficios en microeconomía intermedia. $1^{a}$ Jornada de Docencia de Microeconomía de la Universidad de Granada. 